\title{
Signature of effective mass in crackling-noise asymmetry
}

\section{STEFANO ZAPPERI ${ }^{1,2}$, CLAUDIO CASTELLAN0 ${ }^{1,2 *}$, FRANCESCA COLAIORI ${ }^{1,2}$ AND GIANFRANCO DURIN ${ }^{3}$}

\author{
${ }^{1}$ CNR-INFM SMC, Dipartimento di Fisica, Università “La Sapienza”, P.le A. Moro 2, 00185 Roma, Italy \\ ${ }^{2}$ Istituto dei Sistemi Complessi, CNR, Via dei Taurini 9, 00185 Roma, Italy \\ ${ }^{3}$ IEN Galileo Ferraris and Istituto Nazionale di Ricerca Metrologica, str. delle Cacce 91, 10135 Torino, Italy \\ *e-mail: castella@pil.phys.uniroma1.it
}

C rackling noise is a common feature in many dynamic systems $^{1-9}$, the most familiar instance of which is the sound made by a sheet of paper when crumpled into a ball. Although seemingly random, this noise contains fundamental information about the properties of the system in which it occurs. One potential source of such information lies in the asymmetric shape of noise pulses emitted by a diverse range of noisy systems ${ }^{8-12}$, but the cause of this asymmetry has lacked explanation ${ }^{1}$. Here we show that the leftward asymmetry observed in the Barkhausen effect ${ }^{2}$ - the noise generated by the jerky motion of domain walls as they interact with impurities in a soft magnet-is a direct consequence of a magnetic domain wall's negative effective mass. As well as providing a means of determining domain-wall effective mass from a magnet's Barkhausen noise, our work suggests an inertial explanation for the origin of avalanche asymmetries in crackling-noise phenomena more generally.

Crackling noise is the response of many physical systems to a slow external driving force, characterized by outbursts of activity (avalanches or pulses) spanning a broad range of sizes, separated by quiescent intervals ${ }^{1}$. In condensed matter, notable examples are the magnetization noise emitted along the hysteresis loop in ferromagnets (that is, the Barkhausen effect ${ }^{2}$ ), the noise from magnetic vortices in type-II superconductors ${ }^{3}$, ferroelectric materials ${ }^{4}$ and driven ionic crystals ${ }^{5}$. In the context of mechanics, examples are the acoustic emission signal in fracture ${ }^{6}$ and plasticity ${ }^{7}$ and, on a larger scale, seismic activity corresponding to an earthquake ${ }^{8,9}$. Quantitative understanding of crackling noise is of fundamental importance in different applications, from non-destructive material testing to hazard prediction. This goal can be achieved only through the identification of general universal properties common to these systems, irrespective of their differences in the internal dynamics and microstructural details. In this context, the average shape of the individual pulses of which the signal is composed has been proposed as the best tool to characterize these universal features of crackling noise ${ }^{1}$. In analogy with critical phenomena, it is expected that pulses of different durations can be rescaled on a universal function, whose shape would only depend on general features of the physical process underlying the noise. This scenario is supported by the analysis of a variety of models, where pulse shapes are described by universal symmetric scaling functions ${ }^{12-14}$. In most experimental data, however, the pulse shape is markedly asymmetric with respect to its midpoint, that is, avalanches start quickly but return to zero more slowly ${ }^{1,8-12}$. These results are puzzling because the models accurately reproduce several other universal quantities, such as avalanche distributions and power spectra ${ }^{11,15}$.

One of the most studied examples of crackling noise is the Barkhausen effect recorded in soft magnetic materials. The Barkhausen noise is due to the motion of domain walls, the interfaces separating regions of opposite magnetization, in response to a magnetic field (see the movie in Supplementary Information). Domain walls are characterized by an effective mass ${ }^{16,17}$, which is related to the increase of the wall energy with velocity, as experimentally revealed, for instance, in the dynamic susceptibility of insulating ferrite materials ${ }^{18}$. In metallic ferromagnets, however, inertial effects are usually neglected, as they are much smaller than eddy current dissipation ${ }^{19}$. This approximation is usually assumed in the description of the Barkhausen effect ${ }^{2}$.

We show in this letter that the asymmetry of pulse shapes is a direct signature of the effective mass associated with the objects moving under the action of the external field. In conducting ferromagnets the mass is negative and this results in a leftward asymmetry of the Barkhausen-noise pulses. Our findings clarify the general meaning of pulse shapes in crackling-noise phenomena: the form of the avalanche shows an asymmetry that depends on the duration and encodes important information on the characteristic time of the underlying dynamics. Only on very long timescales are pulse-shape symmetry and universality recovered.

We measure Barkhausen-noise pulse shapes in two ferromagnetic alloys. The experimental setup is depicted in Fig. 1 (see Methods). In response to an increasing external field, domain walls move, inducing in the pick-up coil a voltage signal $v(t)$ proportional to the magnetization rate. The shape of the pulse is defined as the voltage $\langle v(t, T)\rangle$ at time $t$ averaged over all avalanches of duration $T$. When appropriately normalized and 
a
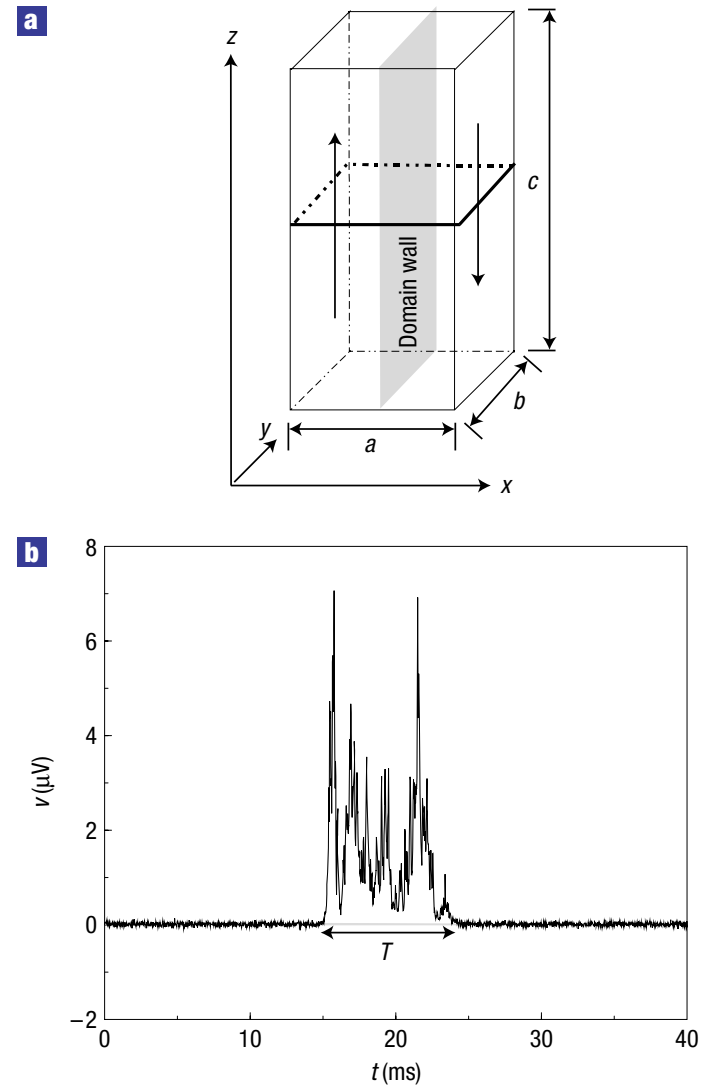

Figure 1 The experimental setup. a, A schematic diagram. A ribbon of dimensions $a \times b \times c$ is placed in a solenoid (not shown) yielding a magnetic field in the vertical direction. The magnetization reversal process occurs through the displacement of domain walls, such as the one depicted here. The arrows indicate the directions of the magnetization. $\mathbf{b}$, An example of the voltage signal $v$, composed of distinct pulses, that is recorded on a pick-up coil wound around the sample.

plotted as a function of $t / T$, the experimentally measured shapes for different durations rescale fairly well (although not perfectly, see later) on a universal function (see Fig. 2a) that is clearly asymmetric, in agreement with earlier measurements ${ }^{10-12}$. Such a leftward asymmetry implies that pulses start rapidly and decay slowly. This is precisely the opposite of the effect of standard inertia, the resistance of a body to changes on its motion, which would imply a slow increase of the velocity when the domain wall is at rest. The asymmetry can be quantified by computing the average skewness

$$
\Sigma(T)=\frac{\frac{1}{T} \int_{0}^{T} \mathrm{~d} t\langle v(t, T)\rangle(t-\bar{t})^{3}}{\left[\frac{1}{T} \int_{0}^{T} \mathrm{~d} t\langle v(t, T)\rangle(t-\bar{t})^{2}\right]^{3 / 2}}
$$

where $\bar{t}=1 / T \int_{0}^{T} \mathrm{~d} t\langle v(t, T)\rangle t$. As shown in Fig. 3, in both samples the skewness is always positive, indicating a leftward asymmetry, and it shows a peak for $T_{\mathrm{p}}^{\exp } \approx 200 \mu$ s.

To account for these experimental results, we make use of a simple and successful model for the Barkhausen effect in soft metallic ferromagnets that is based on the dynamics of a planar domain wall in an effective pinning potential ${ }^{20}$. The equation of motion for the wall position $x$ is given by

$$
\beta \dot{x}=2 I_{\mathrm{s}}\left(c_{H} t-k x+W(x)\right),
$$
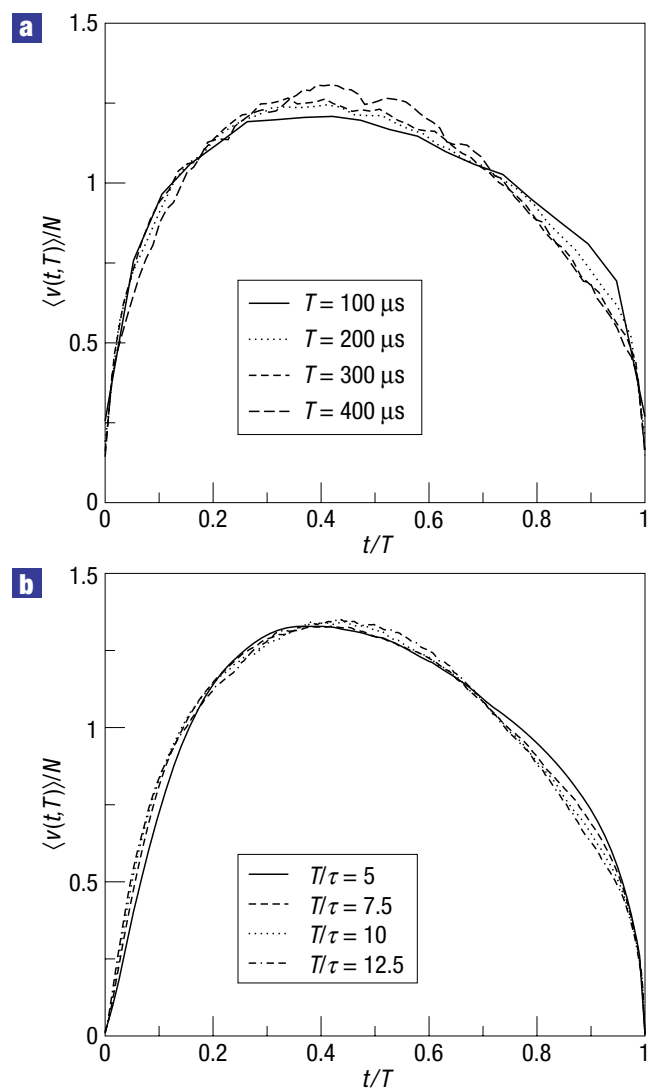

Figure 2 Comparison of the average pulse shapes in the experiments and in the model. a, The average pulse shape obtained from Barkhausen-noise measurements in a partially crystallized $\mathrm{Fe}_{64} \mathrm{CO}_{21} \mathrm{~B}_{15}$ ribbon. The shapes for different durations $T$ are normalized and they rescale quite well, apart from a small systematic variation related to the asymmetry. $\mathbf{b}$, The corresponding shapes obtained from the model. The normalization constant is given by $N=\int_{0}^{T} \mathrm{~d} t\langle v(t, T)\rangle / T$.

where $\beta$ is a damping constant, $I_{\mathrm{s}}$ is the saturation magnetization, $c_{H} t$ is the external field increasing at rate $c_{H},-k x$ is the demagnetizing field and $W(x)$ is a random field with gaussian distribution and brownian correlations ${ }^{20}$. These correlations are believed to represent an effective description of a more general model with flexible domain walls ${ }^{21,22}$. Equation (2) can be solved exactly and provides an excellent description of the statistical properties of the Barkhausen noise, considering that the domainwall velocity $\dot{x}$ is proportional to the recorded $v(t)$. The solution of the model, however, yields a symmetric pulse shape ${ }^{13,14}$, at odds with experimental evidence.

The assumption used to derive equation (2) is that the work done by the effective field (that is, the applied field corrected by the demagnetizing and pinning fields) is compensated by the energy dissipated by eddy currents, which is estimated in the quasistatic approximation ${ }^{20}$. A more detailed analysis of eddy-current dissipation, including dynamic effects (see the Methods section and ref. 23), leads to the identification of a frequency-dependent effective mass, which turns out to be negative in the entire spectrum and equal to $M^{*} \approx-\beta \tau /(2 \gamma)$ at low frequencies, where $\tau$ is the longest relaxation time and $\gamma \approx 1.05$. For the materials considered in our experiments, the effective mass can be estimated to be $M^{*} \approx-7 \times 10^{-5} \mathrm{~kg} \mathrm{~m}^{-2}$, which is much larger than the positive Döring domain-wall mass $\left(M \approx 10^{-9} \mathrm{~kg} \mathrm{~m}^{-2}\right.$; ref. 16). This negative inertial effect can also be formulated by 


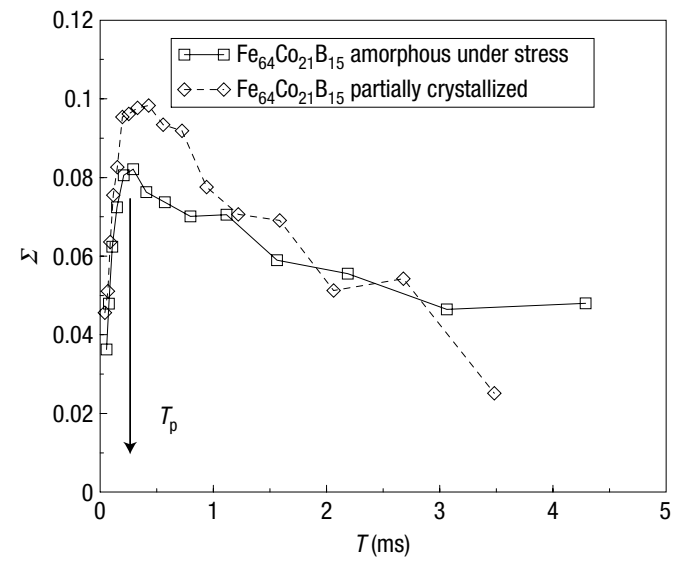

Figure 3 Skewness of avalanches in experiments. The skewness of the pulse shape as a function of the pulse duration obtained from experiments. The data show a peak at $T_{\mathrm{p}}^{\mathrm{exp}} \simeq 200 \mu \mathrm{s}$ indicated by an arrow.

adding a non-local damping term in the equation of motion for the wall (see Methods):

$$
\Gamma \dot{x}+\frac{\Gamma_{0}}{\tau} \int^{t} \mathrm{e}^{-\left(t-t^{\prime}\right) / \tau} \dot{x}\left(t^{\prime}\right) \mathrm{d} t^{\prime}=2 I_{\mathrm{s}}\left(c_{H} t-k x+W(x)\right),
$$

where $\Gamma$ and $\Gamma_{0}$ are coefficients of the same order of magnitude with $\Gamma+\Gamma_{0}=\beta$ and $t^{\prime}$ is an integration (dummy) variable. In order to understand the role of the effective mass on the pulse shape, we have numerically integrated equation (3) for different values of $\tau$. Whereas the distributions of avalanche duration and size are unaffected by the addition of the inertial term, the pulse shapes become asymmetric and bear a remarkable similarity with the experimental ones (see Fig. 2b). Also in this case the skewness is always positive (Fig. 4), indicating a leftward asymmetry, and it shows a peak in correspondence with a characteristic time $T_{\mathrm{p}} \approx 10 \tau$.

Using the value of the largest relaxation timescale for the samples considered experimentally (that is, $\tau=\mu \sigma b^{2} / \pi^{2} \approx 5 \mu \mathrm{s}$, where $b$ is the sample thickness, $\mu$ is the permeability and $\sigma$ is the conductivity), we obtain a theoretical estimate of the peak position $T_{\mathrm{p}} \approx 50 \mu \mathrm{s}$, which is reasonably close to the value measured experimentally, considering the approximations involved in the model. In particular, we have treated a single domain wall moving at the centre of the sample, whereas in the experiments several domain walls are present. This induces a non-trivial interference among the eddy current patterns generated by each wall. To corroborate our interpretation of the asymmetry in terms of a negative effective mass, we have performed simulations of equation (3) replacing the non-local term with a positive mass term $M \ddot{x}$; where $\ddot{x}$ is the second time derivative of $x$ (ref. 24). The resulting skewness, reported in Fig. 4, is negative and shows a peak at $T_{\mathrm{p}} \approx 10 M / \Gamma$.

We have shown that the pulse-shape asymmetry commonly observed in Barkhausen-noise measurements in metals is a signature of a negative effective mass of domain walls. Remarkably, the skewness shows a peak that can be used to track the characteristic relaxation timescale, corresponding to the ratio between mass and damping constant. Similar asymmetric pulse shapes are also observed in seismic data, recorded in correspondence to an earthquake ${ }^{8,9}$. Seismic movements are caused by the motion of fault planes in response to the stress accumulated in the crust. A non-local dynamical effect, analogous to the one discussed

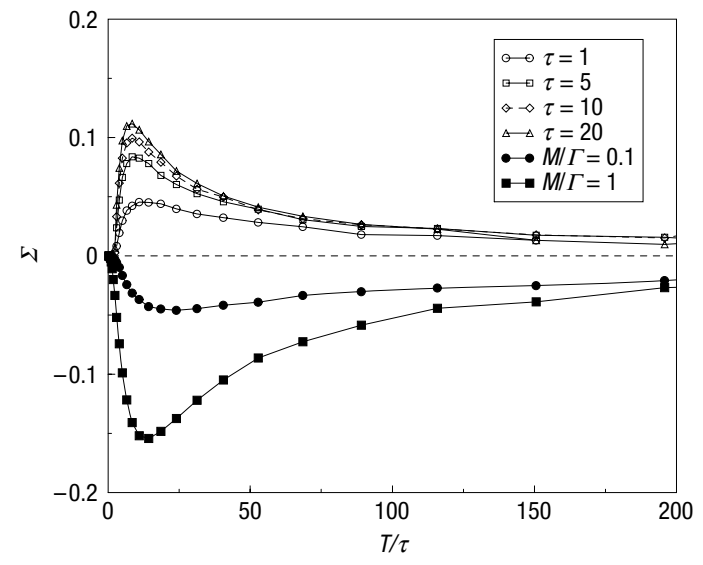

Figure 4 Skewness of avalanches in the model. The skewness of the pulse shape obtained in the domain-wall model for different values of eddy-current time constant $\tau$ is plotted as a function of the pulse duration $T$. We also report a similar measurement in the case of conventional positive domain-wall mass. In the latter case the skewness is negative (rightward asymmetry).

here, could be due to the presence of stress overshoots ${ }^{25}$. It would be interesting to test this mechanism using existing fault-dynamics models.

\section{METHODS}

\section{MATERIALS AND EXPERIMENTAL METHODS}

In our experiments a long solenoid provides a homogeneous driving field $\mathbf{H}$ ramped at constant rate $c_{H}=4 \mathrm{~A} \mathrm{~m}^{-1} \mathrm{~s}^{-1}$ and a secondary pick-up coil around the sample cross-section measures the induced flux. The pick-up coil is made of 50 isolated copper turns, wound within $1 \mathrm{~mm}$. Such a small width is required to avoid spurious effects caused by demagnetizing fields. The measurements are performed only in the central part of the hysteresis loop around the coercive field, where domain-wall motion is the relevant magnetization mechanism. In this case the recorded $v(t)$ is proportional to the domain-wall velocity $\dot{x}$. To reduce excess external noise during the measurement, we use a low-pass pre-amplifier filter with a cutoff frequency of $100 \mathrm{kHz}$. We use two ribbons of $\mathrm{Fe}_{64} \mathrm{Co}_{21} \mathrm{~B}_{15}$, having width $a=1 \mathrm{~cm}$, thickness $b=24 \mu \mathrm{m}$ and length $c=20 \mathrm{~cm}$ : the first sample is amorphous and subjected to a small tensile stress (2 MPa). The second has been partially crystallized (5\%) by thermal annealing. Tensile stress and thermal annealing are used to relax part of the internal stresses, leading to a high signal-to-noise ratio.

\section{DERIVATION OF DOMAIN-WALL MOTION FROM EDDY-CURRENT DISSIPATION}

Considering the configuration described in Fig. 1, the relevant component of field $\mathbf{H}_{\mathrm{e}}=H_{\mathrm{e}}(x, y, t) \hat{z}$, associated with the eddy currents produced by the moving domain wall, obeys the equation ${ }^{19}$

$$
\sigma \mu \frac{\partial H_{\mathrm{e}}}{\partial t}-\nabla^{2} H_{\mathrm{e}}=0 .
$$

One should complement equation (4) with the Faraday condition around the wall

$$
\frac{\partial H_{\mathrm{e}}\left(0^{+}, y, t\right)}{\partial x}-\frac{\partial H_{\mathrm{e}}\left(0^{-}, y, t\right)}{\partial x}=2 \sigma I_{\mathrm{s}} v_{x}(t),
$$

where $v_{x}(t)$ is the domain-wall velocity and we have assumed $c=\infty$. Equations (4) and (5) are solved with the boundary condition $H_{\mathrm{e}}=0$ at the sample surface. The mean pressure on the wall is obtained as

$$
P_{\mathrm{e}}=\frac{2 I_{\mathrm{s}}}{b} \int_{-b / 2}^{b / 2} H_{\mathrm{e}}(0, y, t) \mathrm{d} y=\int \mathrm{d} t^{\prime} f\left(t-t^{\prime}\right) v_{x}\left(t^{\prime}\right) .
$$

A direct calculation of the response function, similar to ref. 23, leads to

$$
f(t)=-\frac{32 I_{s}^{2}}{\mu a \pi^{2}} \theta_{2}\left[\mathrm{e}^{-t / \tau_{a}}\right] \sum_{n=0}^{\infty} \frac{\mathrm{e}^{-t / \tau_{n}}}{(2 n+1)^{2}},
$$

where $\theta_{2}$ is the Jacobi elliptic function and the relaxation times are $\tau_{a}=\mu \sigma a^{2} / 4 \pi^{2}$, and $\tau_{n}=\mu \sigma b^{2} /[(2 n+1) \pi]^{2}$. 
The equation of motion for the domain wall is obtained by equating the eddy current pressure $P_{\mathrm{e}}$ to the pressure $P_{\mathrm{a}}=2 I_{\mathrm{s}} H$ exerted by the applied field corrected by demagnetizing effects and pinning. The left-hand side of equation (3) is recovered by considering the leading contributions at short and long times, involving the largest relaxation time $\tau \equiv \tau_{0}=\mu \sigma b^{2} / \pi^{2}$. The constants can be estimated as

$$
\Gamma=\frac{16 I_{\mathrm{s}}^{2} \sigma b}{\pi^{3}}\left(\gamma-\frac{2 \alpha}{\pi}\right) \quad \Gamma_{0}=\frac{32 I_{\mathrm{s}}^{2} \alpha \sigma b}{\pi^{4}}
$$

with $\alpha \approx 0.886$ and $\gamma=\sum_{n=0}^{\infty} 1 /(2 n+1)^{3} \approx 1.05$.

In the frequency domain, we can formally rewrite equation (6) as $\tilde{P}_{\mathrm{e}}=\left(\beta+i \omega M^{*}\right) \tilde{v}_{x}$, with the effective mass, in the limit of low frequency, $\omega$, given by

$$
M^{*} \approx-\frac{8 I_{\mathrm{s}}^{2} b^{3} \mu \sigma^{2}}{\pi^{5}}=-\frac{\beta \tau}{2 \gamma} .
$$

Received 17 June 2005; accepted 28 July 2005; published 29 September 2005.

References

1. Sethna, J., Dahmen, K. A. \& Myers, C. R. Crackling noise. Nature 410, $242-244$ (2001).

2. Durin, G. \& Zapperi, S. in The Science of Hysteresis (eds Bertotti, G. \& Mayergoyz, I.) 181-267 (Academic, New York, 2005)

3. Field, S., Witt, J., Nori, F. \& Ling, X. Superconducting vortex avalanches. Phys. Rev. Lett. 74 1206-1209 (1995)

4. Colla, E. V., Chao, L. K. \& Weissman, M. B. Barkhausen noise in a relaxor ferroelectric. Phys. Rev. Lett. 88, 017601 (2002).

5. Mitchell, T. B., Bollinger, J. J., Itano, W. M. \& Dubin, D. H. E. Stick-slip dynamics of a stressed ion crystal. Phys. Rev. Lett. 87, 183001 (2001).

6. Petri, A., Paparo, G., Vespignani, A., Alippi, A. \& Costantini, M. Experimental evidence for critical dynamics in microfracturing processes. Phys. Rev. Lett. 73, 3423-3426 (1994).

7. Miguel, M. C., Vespignani, A., Zapperi, S., Weiss, J. \& Grasso, J. R. Intermittent dislocation flow in viscoplastic deformation. Nature 410, 667-670 (2001).

8. Houston, H., Benz, H. M. \& Vidale, J. E. J. Geophys. Res. 103, 29895-29913 (1998).

9. Mehta, A. P., Dahmen, K. A. \& Ben-Zion, Y. Universal shape profiles of earthquake ruptures. cond-mat/0403567 (2004).
10. Spasojevic, D., Bukvic, S., Milosevic, S. \& Stanley, H. E. Barkhausen noise: elementary signals, power laws, and scaling relations. Phys. Rev. E 54, 2531-2546 (1996).

11. Durin, G. \& Zapperi, S. On the power spectrum of magnetization noise. J. Magn. Magn. Mater. 242-245, 1085-1088 (2002).

12. Mehta, A., Mills, A., Dahmen, K. \& Sethna, J. Universal pulse shape scaling function and exponents: a critical test for avalanche models applied to Barkhausen noise. Phys. Rev. E 65, 046139 (2002).

13. O'Brien, K. P. \& Weissman, M. B. Statistical characterization of Barkhausen noise. Phys. Rev. E 50, 3446-3452 (1994).

14. Colaiori, F., Durin, G. \& Zapperi, S. Shape of a Barkhausen pulse. J. Magn. Magn. Mater. 272-276, E533-E534 (2004)

15. Durin, G. \& Zapperi, S. Scaling exponents for Barkhausen avalanches in polycrystalline and amorphous ferromagnets. Phys. Rev. Lett. 84, 4075-4078 (2000).

16. Döring, W. Über die tragheit der wände zwischen weisschen bezirken. Z. Naturforsch. a 3 , 373-379 (1948).

17. Hubert, A. \& Schäfer, R. Magnetic Domains (Springer, New York, 1998).

18. Rado, G. T., Wright, R. W. \& Emerson, W. H. Ferromagnetism at very high frequencies. iii. Two mechanisms of dispersion in a ferrite. Phys. Rev. 80, 273-280 (1950).

19. Bertotti, G. Hysteresis in Magnetism (Academic, San Diego, 1998).

20. Alessandro, B., Beatrice, C., Bertotti, G. \& Montorsi, A. Domain wall dynamics and Barkhausen effect in metallic ferromagnetic materials. i. Theory. J. Appl. Phys. 68, 2901-2908 (1990)

21. Zapperi, S., Cizeau, P., Durin, G. \& Stanley, H. E. Dynamics of a ferromagnetic domain wall: avalanches, depinning transition and the Barkhausen effect. Phys. Rev. B 58, 6353-6366 (1998).

22. Mills, A. C., Hess, F. M. \& Weissman, M. B. Statistics of the pinning field in a soft metallic ferromagnet. Phys. Rev. B 66, 140409(R) (2002).

23. Bishop, J. E. L. The contribution made by eddy currents to the effective mass of a magnetic domain wall. J. Phys. D 13, L15-L19 (1980).

24. Baldassarri, A., Colaiori, F. \& Castellano, C. The average shape of a fluctuation: universality in excursions of stochastic processes. Phys. Rev. Lett. 90, 060601 (2003).

25. Schwarz, J. M. \& Fisher, D. S. Depinning with dynamic stress overshoots: Mean field theory. Phys. Rev. Lett. 87, 096107 (2001).

\section{Acknowledgements}

Correspondence and requests for materials should be addressed to C.C.

Supplementary Information accompanies this paper on www.nature.com/naturephysics.

Competing financial interests

The authors declare that they have no competing financial interests.

Reprints and permission information is available online at http://npg.nature.com/reprintsandpermissions/ 